\title{
Non-circular crane rail theory and parametric design
}

\author{
Chen J.L.*, Dong D.S., Qiao Z. \\ Logistics Engineering College Shanghai Maritime University \\ Email: cjlrock@163.com
}

\begin{abstract}
Rail crane often needs to run on the curve track. Traditional design adopts concentric circle method and handdrawing, but this will take time and effort without reasonable result, consequently, a new idea for design has to be implemented from the beginning. The paper modifies the track theory, with the introduction of clothiod spiral and computing program. The modification leads to the realization of parametric design as well as avoiding defect caused by traditional concentric circle theory. Eventually, the reasonability is verified by the Adams simulation.
\end{abstract}

Keywords: Clothoid Spiral, Rail Theory, Parametric Design, Adams Simulation.

\section{INTRODUCTION}

Crane rail is determined by all wheels under four legs. Additionally, it is impossible to obtain an inner or outer track through monolithic moving from center line for the gauge of crane, which is much wider than that of ordinary vehicles. Therefore, the method of designing these tracks is to assume an inner or outer track firstly, then calculate the other track correspondingly, finally, generate two different and related tracks. The basic principle of crane rail is studied, and there exists a design approach based on the modification of both track itself and wheel tread [1]. From the application point of view, related results of circular track, such as the minimum radius of curvature, wheel-rail clearance and amount of derailment are calculated [2,3]. Traditional the basic principle of circular track, radius of curvature leaping from infinity to a certain numerical value, leads to a remarkable increase of the amount of derailment even exceeds the tolerance value. Fatally, this can contribute to damage and destruction on rails and cranes. Since circular tracks on longer meet the satisfactions of practical requirements, the import of noncircular track is of great necessary. Although there is not much related research in crane, the problem had long been discussed in road and railway design. The coordinate calculation formula of clothoid spiral is deduced and flexible applied in road and railway design by the means of AutoCAD embedded programming languages [4]. As the clothoid spiral is fairly complicated to use especially in programming, some approximate methods are implemented to approach the clothoid spiral [5,6]. Furthermore, fast local curve interpolation algorithms are rapidly developed for the purpose of smoothening, fitting and mixing all kinds of curves [7]. The paper takes the corner rail as the research objection studies the generation method and correction principle of non-circular rails, specifically, clothoid spiral. Also a parametric design is realized by MATLAB and the accuracy of the program is verified by ADAMS simulation.

\section{NON-CIRCULAR RAIL THEORY}

\subsection{Selection and calculation of non-circular rail}

As the inner rail is a basic non-circular rail, the transition curve which is inserted between straight line and circle changes variedly. The clothoid spiral is one among all kinds of transition curves. The clothoid spiral is also known in mathematics as Eular Spiral, which is raised by Euler in 1744 while studying the free tension at both ends of the elastic spring tension problem. Cornu suggests a more precise description of the spiral in 1874, therefore Eular Spiral is also known as Cornu Spiral. The Euler Spiral is a family of curves whose curvature changing with respect to the arc length of the curve is linear. Generally speaking, the curvature of such curves is continuous along the length of the curve. With such good geometric properties, Eular Spiral is widely used in the field of engineering, E.g. railway track design, highway corners design, computer animation path design, vector font design, etc.

The expression for the clothoid spiral is $\rho l=C, C$ is a real constant. Apparently, the expression cannot be calculated directly and need to be transformed into parametric equations. Central angle (helix angle) which is corresponding to the arc length between the point $\mathrm{P}$ on the clothoid spiral and the start point of the spiral point $O_{1}$, and from the geometric 
relationship we can also see central angle is angle between the tangent of the point $\mathrm{P}$ and that of start point $O_{1}$. So if we take a differential arc $d_{l}$ at point $\mathrm{P}$, its corresponding central angle is $d \beta=\frac{d_{l}}{\rho}=\frac{l \cdot d_{l}}{c}=\frac{l d_{l}}{R_{2} L s}$, solve the integral and it will be $\beta=\int_{0}^{l} \frac{u}{R_{2} L s} d u=\frac{l^{2}}{2 R_{2} L s}$. Taken $O_{1}$ as the origin of coordinates, the tangent through $O_{1}$ is the $\mathrm{X}$ axis, the direction of the radius is the $\mathrm{Y}$ axis, so the coordinate of $\mathrm{P}$ is $\mathrm{x}$ and $\mathrm{y}$, the projected length of $d_{l}$ on the coordinate axis is $d_{x}$ and $d_{y}$, respectively. So

$\left\{\begin{array}{l}d_{x}=d_{l} \cdot \cos \beta \\ d_{y}=d_{l} \cdot \sin \beta\end{array}\right.$

Applying Taylor series expansion to $\cos \beta$ and $\sin \beta$ on zero to the forth and substituted in equation (1) and solve the integral

$$
\left\{\begin{array}{l}
x=l-\frac{l^{5}}{40 c^{2}}+\frac{l^{9}}{3456 c^{4}}-\frac{l^{13}}{599040 c^{6}} \\
y=-\left(\frac{l^{3}}{6 c}-\frac{l^{7}}{336 c^{3}}+\frac{l^{11}}{42240 c^{5}}-\frac{l^{15}}{1612800 c^{7}}\right)
\end{array}\right.
$$

where $c=R_{0} \cdot L_{S}$, all parameter function in the paper is expressed by arc length $l$. The track of inner rail consists of straight line; clothoid spiral and circle (see Fig. 1.). The expression of piecewise function as

$$
\begin{aligned}
& \begin{cases}x_{1}=l & l \leq 0 \\
y_{1}=0 & l \leq 0\end{cases} \\
& \begin{cases}x_{1}=l-\frac{l^{5}}{40 c^{2}}+\frac{l^{9}}{3456 c^{4}}-\frac{l^{13}}{599040 c^{6}} & 0<l \leq L_{S} \\
y_{1}=-\left(\frac{l^{3}}{6 c}-\frac{l^{7}}{336 c^{3}}+\frac{l^{11}}{42240 c^{5}}-\frac{l^{15}}{1612800 c^{7}}\right) & 0<l \leq L_{S}\end{cases} \\
& \begin{cases}x_{1}=R_{0} \cdot \sin \left(\beta_{0}+\frac{l-L_{S}}{R_{0}}\right)+\mathrm{x}_{0} & L_{S}<l \\
y_{1}=R_{0} \cdot \cos \left(\beta_{0}+\frac{l-L_{S}}{R_{0}}\right)+\mathrm{y}_{0} & L_{S}<l\end{cases}
\end{aligned}
$$

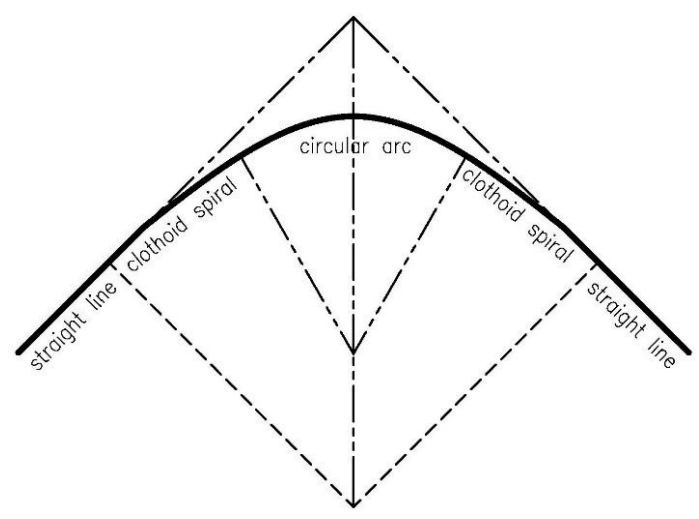

Figure 1. Inner curve schematic diagram where $\beta_{0}=L_{S} / 2 R_{0}$ is the angle of contingence which is joint of clothoid spiral and circle, $\left(x_{0}, \mathrm{y}_{0}\right)$ is the coordinates of center of circle, $x_{0}=L_{S}-\frac{L_{S}^{3}}{40 R_{0}^{2}}+\frac{L_{S}^{5}}{3456 R_{0}^{4}}-\frac{L_{S}^{7}}{599040 R_{0}^{6}}$, $y_{0}=-\left(\frac{L_{S}^{2}}{6 R_{0}}-\frac{L_{S}^{4}}{336 R_{0}^{3}}+\frac{L_{S}^{6}}{42240 R_{0}^{5}}-\frac{L_{S}^{8}}{1612800 R_{0}^{7}}\right)$.

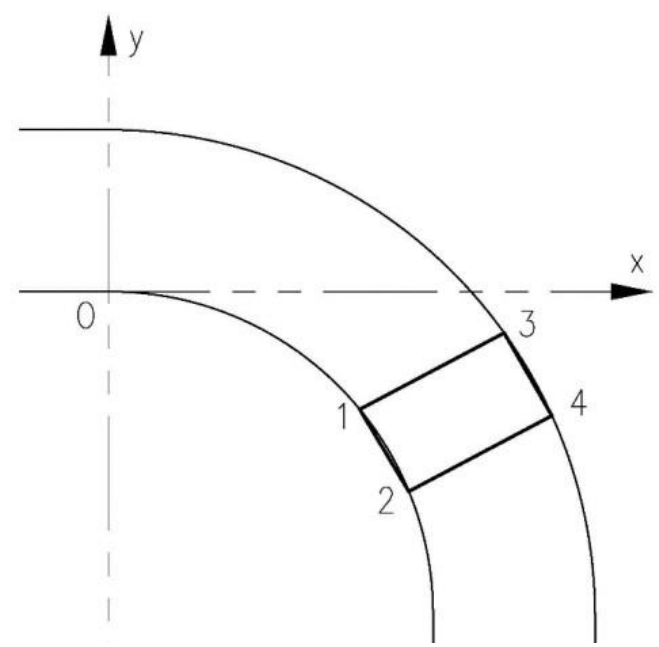

Figure 2. Leg frame schematic diagram

As the point 1 and 2 under two crane legs move severely through the inner rail, the track of point 3 and 4 is outer rail by a sequence of operation (see Fig. 2.). The equation of point 3 as

$\left\{\begin{array}{l}x_{3}(l)=-k_{12}(l) \cdot \sqrt{\frac{S^{2}}{1+k_{12}^{2}(l)}}+x_{1}(l) \\ y_{3}(l)=\sqrt{\frac{S^{2}}{1+k_{12}^{2}(l)}+y_{1}(l)}\end{array}\right.$

Similarly, the equation of point 4 is similar as that of point 3 as

$\left\{\begin{array}{l}x_{4}(l)=-k_{12}(l) \cdot \sqrt{\frac{S^{2}}{1+k_{12}^{2}(l)}}+x_{2}(l) \\ y_{4}(l)=\sqrt{\frac{S^{2}}{1+k_{12}^{2}(l)}}+y_{2}(l)\end{array}\right.$

There exists an excursion between point 3 and 4, judging from Eq. (4) and (5), so the final outer rail is the mid-point track between point 3 and 4 through interpolation and the excursion between point 3 and 4 is inevitable.

\subsection{Modification theory of non-circular rail}

The theory mentioned above is only suitable for single wheel but the reality is that there always a couple of wheels under one leg, so the modification of multi-wheel is necessary. Practically, wheels never change their direction until one wheel come into contact with the rail. Wheels go straight forward before contact with the rail, after that wheel rotates a certain degree and go straight again. This process is 
continuously performed during the period of turning a corner. Obviously, the realization of this process is rather complex. So, the theoretical calculation could not be the same as such progress, therefore we need a simplified theory to modify multi-wheel tracks.

Here we assumes that the distance from both sides of wheel edges to the center line is the same (see Fig. 3). Consequently, the distance between the rail center and the wheel set center is named as the deviation value $\delta$.

$\delta=\sqrt{x_{A}^{2}+\left(d+R_{0}-y_{A}\right)^{2}}-R_{0}-\frac{B_{1}}{2}=R_{0}-\frac{B_{1}}{2}-\sqrt{x_{B}^{2}+\left(d+R_{0}-y_{B}\right)^{2}}$

where $\delta$ and $d$ both are unknown numbers, the value of $d$ can be obtained by solving the right two functions. Thus $\delta$ will also get a corresponding value.

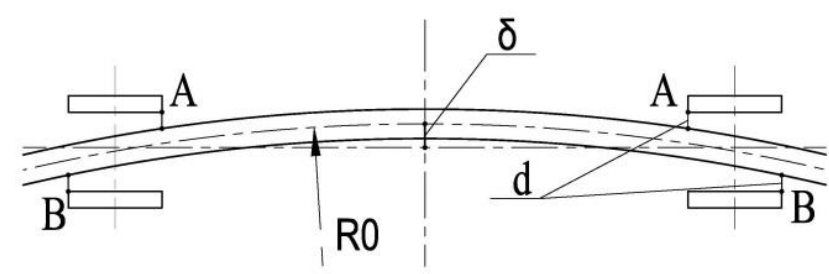

Figure 3. Deviation value schematic diagram

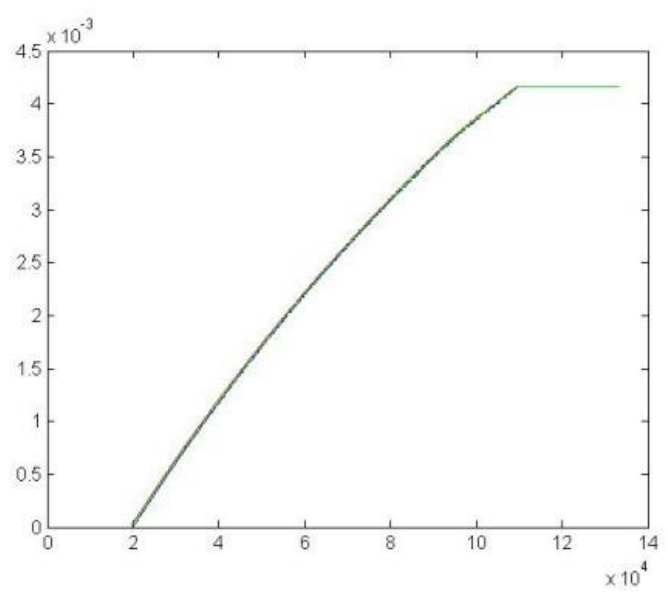

Figure 4. Deviation value result

As is described in E.q (6), deviation value $\delta$ is related with the radius of circle, so the deviation value varies with radius during the clothoid spiral. So, the variation trend of the modification value $\delta$ needs to be confirmed during the clothoid spiral. After calculating the deviation value of one whole rail (see Fig. 4), it is obviously that there are three different lines. The first one from 0 to 2 is the deviation value of straight lines. The second bias is that of the clothoid spiral. The last straight line around between 10 and 12 is the circle. What we want is the tendency of the second line, which is a clothoid spiral, the relationship between deviation value and arc length is approximately linear. So we assume that the approximate equivalent of the deviation value during the clothoid spiral is that the deviation value is a linear distribution along the arc length.

The above discussion is the two-wheel situation. As to cranes, the wheel number is usually more than two, even up to twelve, while the manufactory of wheels are based on twowheel modules. Take an eight-wheel as example (see Fig. 5), the total deviation value $\delta$ is composed by three parts (see Fig. 6). $\delta_{1}$ is the deviation value of $\mathrm{K} 1$, so are $\delta 2$ and $\delta$ 3.Although the direction of $\delta_{1}, \delta_{2}$ and $\delta_{3}$ is different, the actual angle difference is very low only about $0.01^{\circ}$, so the accumulation is feasible as

$\delta=\sum_{i=1}^{n} \delta_{i} \quad(i=1,2,3)$

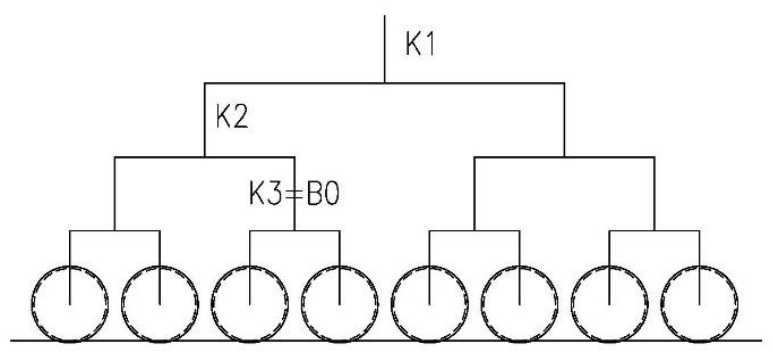

Figure 5. Eight-wheel distribution

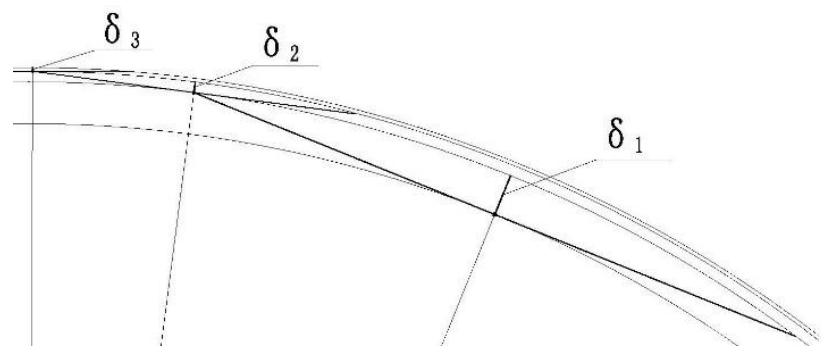

Figure 6. Eight-wheel deviation value

\section{PARAMENTRIC DESIGN}

\subsection{Matlab}

Matlab is the commercial mathematics software produced by American MathWorks Company. With its powerful function in scientific calculation and mathematical analysis, Matlab has become one of the most popular scientific engineering software in mathematics. Through its years of efforts, MathWorks has developed a suite of toolkits for control system applications, signal processing, graphics processing, communications, finance and finance, etc. At present, MATLAB have been widely used in aviation, aerospace, automotive, communications and other industries, and gradually become one of the best software applications in industry.

\subsection{Parametric design based on MATLAB}

Through coding all theory mentioned above, the parametric design can be realized by MATLAB. The program is divided into two main segment which is singlewheel generation and multi-wheel modification. The singlewheel generation is responsible for creating a track according to input data and the function of multi-wheel modification is to calculate the deviation value to amend the track that just created.

Also there is a simple GUI Windows operating system (see Fig. 7) for users. Users only need to use Windows operation system without any input code to achieve parametric design. 
In the process of parametric design, the input data is radius, arc length, base distance, gauge, turning angle and wheel number. Based on these input data, the program will output the inner and outer track (see Fig. 8) as well as related coordinate points in text document.

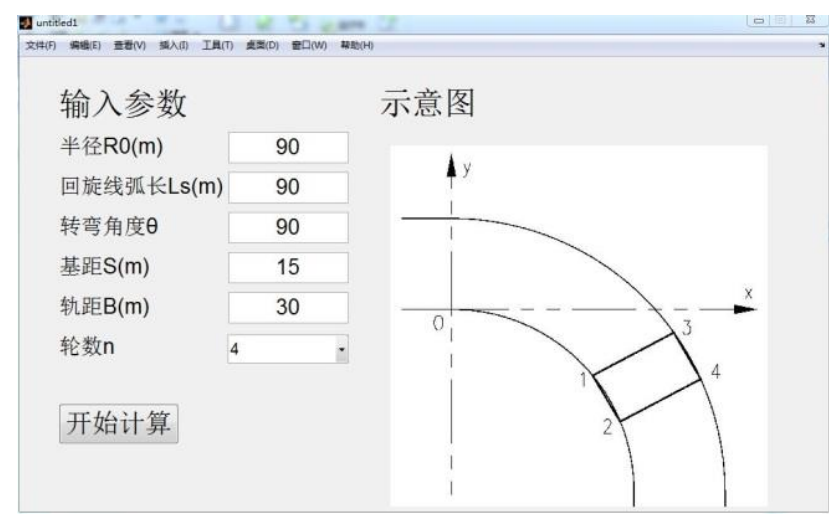

Figure 7. GUI Windows

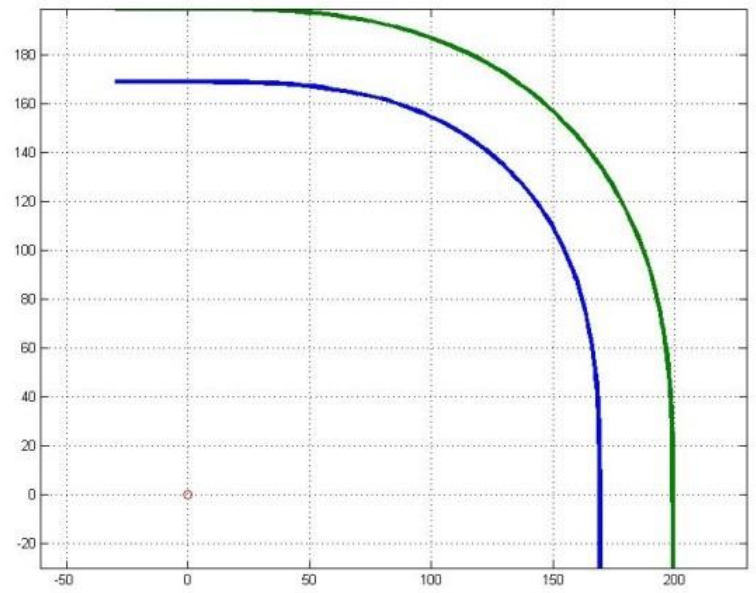

Figure 8. Final rail track

\section{ADAMS SIMULATION}

\subsection{ADAMS}

ADAMS is a multi-body dynamics simulation software equipped with Fortran and $\mathrm{C}++$ numerical solvers. ADAMS was originally developed by Mechanical Dynamics Incorporation which then was acquired by MSC Software Corporation. It can be used to create fully parametric mechanical system geometry and analyze the virtual machine's system statics, kinematics and dynamics status, through establishing the dynamic equations, to output displacement, velocity, acceleration, etc. Its solver is based on the Lagrangian equation method in the dynamics theory of multiple rigid body systems. ADAMS can also simulate the performance of the mechanical system, motion range, collision detection, peak load and others.

\subsection{Simulation results}

The ADAMS simulation can read the data from files like txt and excel, so any track transformed into these two data can be imported into ADAMS if for simulation, in order to verify whether the track is reasonable or not. All tracks generated by MATLAB can be verified by ADAMS simulation.

There is an excursion between two outer wheels through the research on theory. The result of theory (see Fig. 9a) and simulation (see Fig. 9b) are excursions of one complete track based on same input data. Judging from the figures, the result is rather approximate. A more precise result (see Tab.1) shows that the error between theory and simulation is very small and the error decreases while the length of clothoid spiral increases. Therefore, although there are some partial simplifications and approximations in the theory, the error is absolutely small within tolerance while the theory excursion is always lower than the simulation excursion.

Table 1. Comparison table of theory and simulation

\begin{tabular}{llllll}
\hline \multicolumn{2}{l}{ Length of clothoid spiral $(\mathrm{m})$} & 30 & 60 & 90 & 120 \\
\hline $\begin{array}{l}\text { Deviation } \\
\text { value }(\mathrm{mm})\end{array}$ & Theory & 28 & 15.7 & 10.8 & 8.2 \\
\cline { 2 - 6 } & Simulation & 28.6 & 15.8 & 10.9 & 8.3 \\
\hline
\end{tabular}

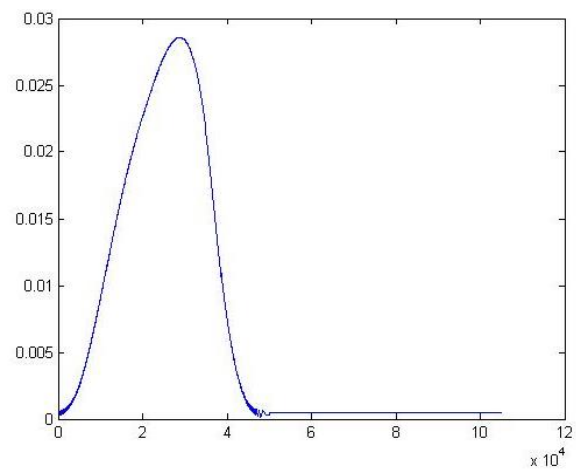

Figure 9a. Excursion of theory

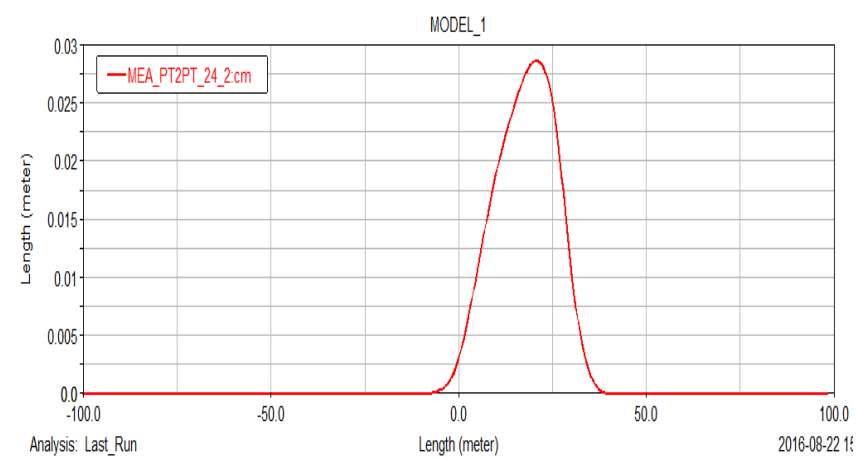

Figure 9b. Excursion of simulation

\section{CONCLUSIONS}

This is the first time we introduce the clothoid spiral as a transition curve in crane rail design, and the result is undoubtedly reasonable.

For the purpose of parametric design, the non-circular rail theory is separated into two part, single-wheel and multiwheel. The segregation of two parts makes the computing programming possible and avoids problems encountered in the past. The final excursion is composed by that of singlewheel and multi-wheel. The single-wheel accounts for the majority of all. As to multi-wheel, since it has a much less 
influence on the final excursion, we introduced some assumptions which is linearization and direct accumulation for the purpose of parametric design and computer programming. Despite such simplifications and approximations, the result of simulation verifies the rationality of these simplification and approximations while the result of theory is a little lower than that of simulation. Furthermore, it is worth mention that the shorter the clothoid spiral length is, the larger the error between theory and simulation is.

\section{REFERENCES}

[1] Ni X.Q. (1980). The turning problem of rail crane, Lifting the Transport Machinery, pp. 69-79.

[2] Xu G.L. (1995). Discussion on turning problem of crane, Construction Machinery, pp. 8-12.

[3] Wang W.T. (2008). Design of large rail crane turning, Chinese Journal of Construction Machinery, Vol. 2, pp. 87-90.

[4] Zhu H.P., Wang W. (2010). Algorithm and drawing of clothoid spiral in road design, Urban Roads Bridges \& Flood Control, Vol. 1, pp. 23-25.

[5] Walton D.J., Meek D.S. (2005). A controlled clothoid spline, Computers \& Graphics, Vol. 29, No. 3, pp. 353-363.

[6] Mccrae J., Singh K. (2009). Sketching piecewise clothoid curves, Computers \& Graphics, Vol. 33, No. 4, pp. 452-461.

[7] Havemann S., Edelsbrunner, J., Wagner P., et al. (2013). Curvature-controlled curve editing using

piecewise clothoid curves, Computers \& Graphics, Vol. 37, No. 6, pp. 764-773.

[8] Sun Y.G., Qiang H.Y., Chang D.F., Wang R. (2016). Response characteristic analysis of nonlinear vortexinduced vibration of tension leg platform in deep sea, Journal of the Balkan Tribological Association, Vol. 22, No. 3, pp. 2519-2536.

[9] Sun Y. G., Li W.L., Dong D. S., Mei X., Qiang H. Y. (2015). Dynamics analysis and active control of a floating crane, Tehnicki Vjesnik-Technical Gazette, Vol. 22, No. 6, pp. 1383-1391.

\section{NOMENCLATURE}

1

$\mathrm{O}_{1}$

$\mathrm{d}_{1}$

$\mathrm{d}_{\mathrm{x}}$

$\mathrm{d}_{\mathrm{y}}$

$\mathrm{R}_{0}$

$\mathrm{L}_{\mathrm{S}}$

$\mathrm{k}_{12}$

d

$\mathrm{B}_{1}$

\section{Greek symbols}

$\rho$

$\beta$ arc length of clothoid spiral start point of the spiral point differential arc

differential arc on $\mathrm{X}$ axis differential arc on $\mathrm{Y}$ axis radius of clothoid spiral total clothoid spiral length slope of line across point 1 and 2 distance from both sides of wheel edges width of rail

curvature radius central angle (helix angle) deviation value 